\title{
Improved Image Denoising Methodology using Deep CNN Bilateral Filter Compared to Additional Methods
}

\author{
Pallavi Bora, Kapil Chaudhary
}

\begin{abstract}
Image Denoising techniques are widely used to remove the noise from the images. Due to the ease of the bilateral filter, it is used very often to remove the noise from the images. In this paper, a novel approach has been proposed to enhanced bilateral filter in conjunction with CNN as a booster to eliminate Gaussian noise from Grey images. Studies reveal that standard CNN using a bilateral filter is the best technique to eliminate Gaussian noise from images along with high PSNR values. This paper also performs a comparative study of the various existing techniques for image denoising with the CNN technique and the applied Bilateral filter Method as a de facto to improve the results in terms of enhanced PSNR values. ECNDNet (Enhanced CNN) applied to noisy images with standard deviation $\sigma=15$ gives PSNR values up to 32.81 In comparison to this when both bilateral filter and deep CNN applied, in conjunction produces improved PSNR values up to 34.73 along with the equivalent standard deviation. The results in this work reveal better performance in terms of PSNR as compared to other methods. The test result proves that the bilateral filter Method along with CNN can improve the quality of restored images significantly better.
\end{abstract}

Keywords: Image Denoising, PSNR, Bilateral filter, CNN

\section{INTRODUCTION}

Image Denoising is a traditional technique; it has been required in many fields. Images acquired through the sensor of cameras are always corrupted by some noise. The existence of random discrepancy of intensity affects color information images usually can be the feature of electronic noise. The denoising model is the process to eliminate unwanted information from an image, different images are denoised by using different technologies. This research work explains and explores different methodologies for noise reduction. Generally, bilateral filtering makes a linear combination of similar pixel values. Bilateral filtering images depends on range and domain. For domain filtering values choose to show the preferred amount of combination of pixels, while range filtering chooses values based on the preferred amount of wide-ranging pass filtering.

Manuscript received on March 28, 2021.

Revised Manuscript received on June 09, 2021.

Manuscript published on June 30, 2021.

* Correspondence Author

Pallavi Bora*, M-Tech Scholar, Department of Computer Science and Engineering, B.T.K.I.T. Dwarahat, Almora (Uttarakhand), India.

Dr. Kapil Chaudhary, Assistant Professor, Department of Computer Science and Engineering, B.T.K.I.T. Dwarahat, Almora (Uttarakhand), India.

(C) The Authors. Published by Blue Eyes Intelligence Engineering and Sciences Publication (BEIESP). This is an open access article under the CC BY-NC-ND license (http://creativecommons.org/licenses/by-nc-nd/4.0/)
Bilateral filtering technique smooth images while edgepreserving. Its filtering work in 1995 by Aurich and Weule [1] on linear Gaussian filtering.

\section{Advantages of Bilateral Filter:}

- bilateral filtering edge-preserving, while removing noise and causes smoothing of images.

- It reduces color blurriness while working with color images.

- It is a very simple and non-iterative scheme to implement.

The rest of the paper organize in such a manner that, section 2 will briefly introduce related work, section 3 discuss the image denoising techniques, Section 4 will discuss the proposed model, section 5 introduce experiment and data, and also will explain regarding data set Section 6 will explain the result and section 7 will conclude the future scope and conclusion.

\section{RELATED WORK}

In 2017, Kai Zhang et al. [11] presented by exploring the creation of feed-forward denoising with CNN (convolutional neural network). Specifically, this technique is applied to residual learning and batch normalization. It helps speed up the training process. This approach influences training a single DnCNN model. Such as Gaussian denoising JPEG image DE blocking.

In 2017, N. Senthilkumaran, J. Saromary et al [14] proposed an Adaptive Bilateral filtering method which consisted Switched bilateral filter and a bilateral filter to eliminate Gaussian Noise from Gray images and found that bilateral filtering is the best procedure to remove Gaussian noise from images with high PSNR values. The proposed technique gives the best results than all other techniques in terms of assessment parameters such as PSNR, MSE, and NCD.

In 2018[15] Yapeng Tian et.al presented CNN has achieved great success for image restoration also hierarchical features. This approach to generating visually pleasing high-quality measurement. This paper proposed local and global features and obtaining very powerful representation ability.

In 2018,[16] Chunwi Tian, et al. proposed the CNN technique for image denoising by enhancing the quality of various images experimentally and analyzing the PSNR values on different standards deviation. Work demonstrated was looked to the effects of dilated convolution. Its uses for ECND Net to enlarge the receptive fields and improve the performance.
Blue Eyes Intelligence Engineering and Sciences Publication (c) Copyright: All rights reserved.

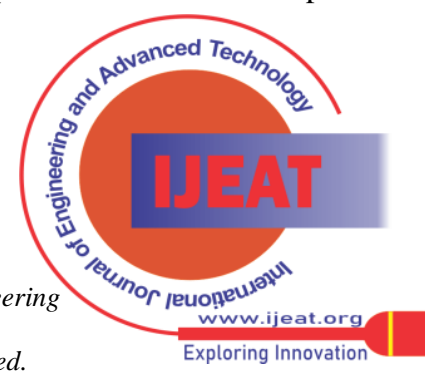


Image restoration[17] techniques developed in the past years and discuss future developments. Learn a CNN to predict the residual between the initialized high-resolution images and the ground truth image. Deep CNN can be very efficient in the testing stage. Image restoration methodologies do not scale well to larger images, while efficient alternative bigot.

\section{IMAGE DENOISING TECHNIQUE}

Bilateral filter denoising [2] improved the images to remove Gaussian noise from the grayscale. It is found the standard Bilateral filter is the best method to eliminate Gaussian noise from images with high PSNR value. Bilateral filter methods are more effective than popular denoising methods. One of the most popular advantages to preserving edges while removing noise causes smoothing of images and reduces color blurriness. It is a simple and iterative scheme to contrivance. Which is a

Stimulating research area that has conventionally use statistical image features. Bilateral has shown recent promise for this task and further improvements can be realized via the use of the PSNR ratio, which allows the use of a full reference quality applied to extracted pixel and original images.

Image restoration [17] essential difficulty in image processing and low-level visualization. Objective reconstructed to latent high-quality image from its ruined reflection. The acquired images are a ruined reflection of the unknown latent images, while the ruin comes from various factors such as noise corruption object motion, resolution limit hazing rain, a combination of them. Introduce the major IR techniques developed in the past years and discuss future developments. Learn a CNN to predict the residual between the initialized high resolution images and the ground truth image. Deep CNN can be very efficient in the testing stage. Image restoration approaches do not scale well to larger images, while efficient alternative. "Goal of this paper is to develop an effective approach to image restored offers both computational efficiency and high restoration quality".

\section{classification}

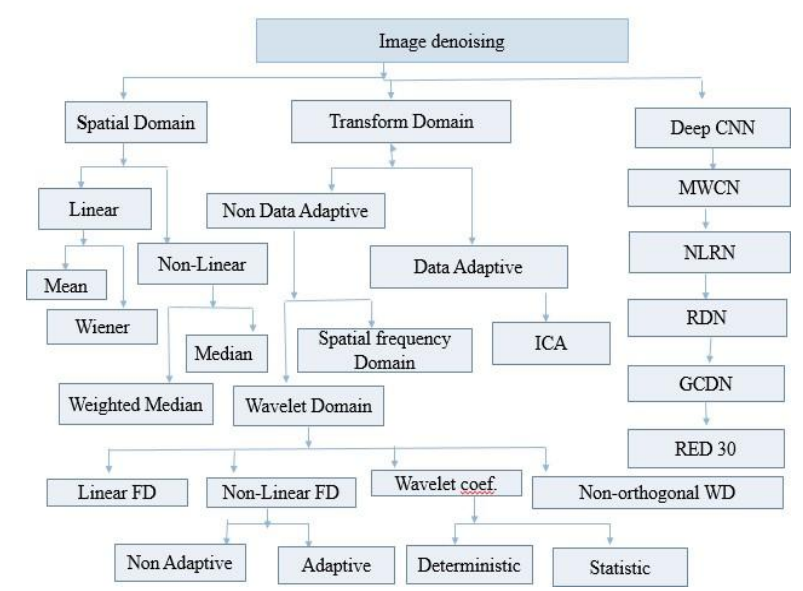

Figure 3.1: Image denoising method

Enhanced CNN [19] used for removing noise in pixel quality better PSNR. This paper used the dilated convolutional networks to increase the perspective information and reduce the computational cost. Enhanced convolutional neural denoising network exceeds the state- of-the-art methods for image denoising. Convolutional neural networks are used to increase network performance. It is used to obtain an uncontaminated image from the residual image and noisy image.

\section{Comparison of Denoising Methods}

This section briefly describes the denoising methods bilateral filtering is reasonable with popular methods suchasBM3D, WNNM, EPLL, CSF, TNRD, FFD, and ECN. WNNM [7] weight nuclear norm minimization problems are evaluated under different conditions. However, this impressively confines its proficiency and flexibility in allocating with many concrete denoising problems. This paper analyzed the weighted nuclear norm minimization problem, where the curious values are allocated different weights. WNNM algorithm outperforms many states of the art denoising algorithm.

EPL [8] expected patch log-likelihood method has been introduced, enforced the concluding reconstructed image patch. In the perspective of the Gaussian mixtures model, this idea has been lead to a state of the art in image denoising and de-blurring. This paper represents a method that essentially concludes these thresholds to improve the image evaluation. It's accomplished a similar performance to that of EPLL with GMM in denoising. TNRD [10] trainable nonlinear reaction-diffusion describes a knowledge agenda built on the perception of nonlinear reaction models for various image restoration problems. This methodology is appropriate for a variety of image restoration tasks by including suitable reaction force. We determine its proficiencies with the representative application, Gaussian image denoising single image super restoration, and JPEG de-blocking. BM3D was developed by Ahn B, et al [13] is the foremost prevalent denoising strategy. BM3D is a two arrange non-locally collaboration sifting strategy within the change space. It utilizes 3d shapes, which are stacked into a 4Dchart. ffD Net [16] is to the fast implication and respectable performance, however, this method mostly learn a specific model for each noise level, require multiple models for denoising images with the different noise level. Fast and flexible denoising network works on accomplishing a good trade-off between implication speed and denoising performance. FFD Net is effective and efficient, making it a highly attractive practical denoising application. CSF [18] shrinkage operation bears a connection to wavelet methodologies, but is used here in a random field context computational proficiency is accomplished by creation concluded the use of convolutional high renewal quality is attained through loss-based training of all models parameters and the use of cascade architecture. We study approaches that can be revised easily to a different trade-off between efficiency and image quality.

\section{PROPOSED DENOISING METHOD}

According to the previous analysis, we all know that the denoising methodology will be expressed as $\mathrm{Y}=\mathrm{x}+\mu$. $\mathrm{F}(\mathrm{y})$, because the objective performance of learning is as follows:-

Published By:

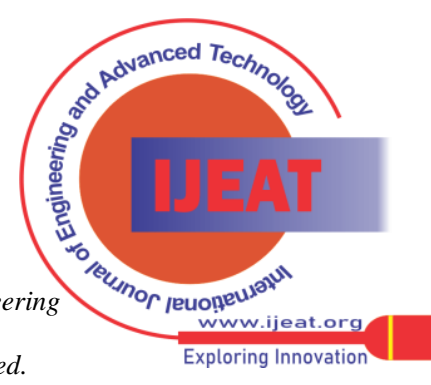




$$
=\frac{1}{-} \sum_{j=1}^{N} \| f\left(y_{j}, p\right)-\left(y_{j}-x_{j}\right)_{\|^{2}}
$$

$\mathrm{N}$

Eq.(1) is an objective function to train the denoising model, where $y_{j}$ represent the $j^{\text {th }}$ noisy image patch and $x_{j}$ represent the $j^{t h l e v e l ~ i m a g e ~ p a t c h ~ a n d ~ l e a r n ~ m o r e ~ f e a t u r e s . ~}$ Learn a lot of possibility as a result of these considerations, we tend to propose a unique network know as Bilateral with CNN furthermore residual learning builds this network more practical for image denoising the deserves of the projected strategies have fielded it in $17^{\text {th }}$ layer network and residual learning to stop the issues of vanishing a further CNN performance convergence and build the network easier to trained knowledge. Moreover, the use of $\mathrm{BN}$ and residual learning make this network more effective for image denoising. In addition to bilateral filter with CNN enhanced performance $\mathrm{BN}$ techniques are used to convergence and make the network easier to trained data.

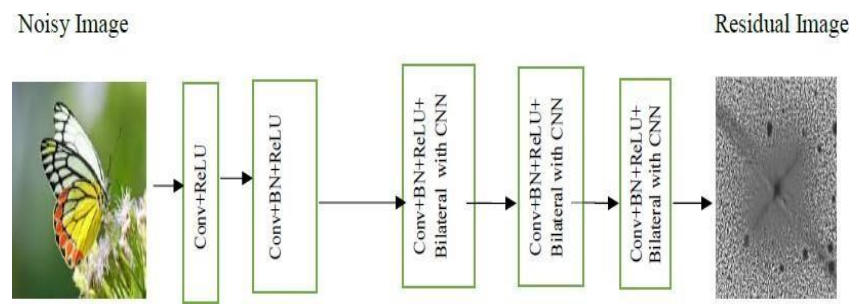

The Bilateral filtering technique using CNN analysis consists of stages:-

1 Basic original images to differentiate the denoised from noised level from the CNN.

2 Adaptive iteration use frequently changes PSNR noise and pixel similarity analysis to visually match the denoised with the original images.

3 At last, the images which denoised Bilateral filter using CNN technique.

This approach is very convenient for robustness and helps in improving the PSNR to survive against different types of techniques, also by using CNN. The extraction process involves increasing the pixel quality of denoised images.

\section{Application of Bilateral Filtering:}

- Bilateral filter, wherever it found a broad application that medical imaging pursuit film restoration and a lot of we tend to discuss helpful extension referred to as the bilateral filter.

- A bilateral filter could be a feature formula the discrepancy will be used to extend the visibility of alternative pictures searching filters that train to scale back noise a large style of ultimate. Edge sharpening filters company by enhancing high frequency.

- Bilateral filtering application to decompose many pictures into parts so they single output image that inherits selected visual properties from every picture.

- We will additionally utilize the assorted technique like texture and illumination separation tendency creating and residency management for bilateral filtering.

\section{DATASET \& IMPLEMENTATION:}

This paper technique is enforced Matlab-19 and varied performance metrics are unit taken into thought peak signal
Figure 4.1: Architecture of Proposed method

noise magnitude relation a good picture quality of denoising images. This study aims to evaluate the working, performance, and efficiency of various denoising techniques. This study helps in knowing different types of techniques used to remove noise in original images. Data set used for experimental purpose as follows:

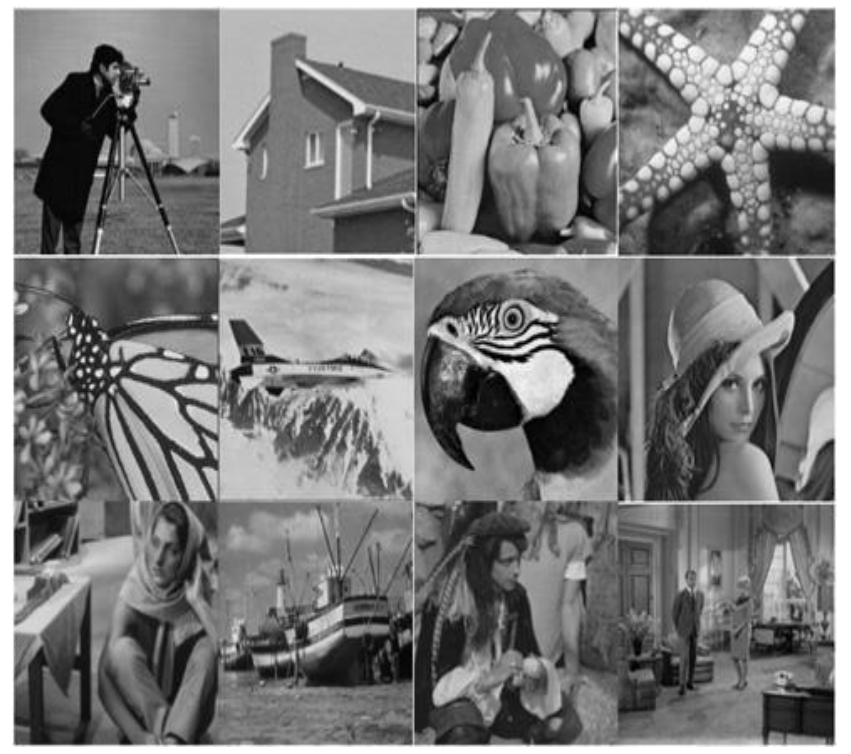

Figure 5.1:

For other experimental, this paper used various techniques such as a bilateral filter using CNN. The experimental results of this technique are well tried to point out that its capable of image denoising single image superresolution and JPEG in image artifacts removal. Above all the images throughout Gray pictures, sp compared to the bilateral filtering methodology and bilateral with CNN preexisting denoising methodology result. lts promising within the sense that was ready to recover images details in structures within the actual ensuring image was a lot of visual images.

We tend to noise magnitude relation PSNR of each image. The $\mathrm{BN}$ and residual learning are effective for image denoising. We all know that the bilateral filter with CNN is useful for image denoising.

Bilateral for Grey images denoising: We choose 12 pictures with the dimensions of $180 * 180$ for mathematicians denoising the format of pictures is png. In line with BM3D, WNNM, EPPL, CSF, TNDR, IRCNN, ECNDNet, and bilateral with CNN to verify the performance of gray pictures. Denoising to check the strength of our project methodology for low-level and high-level noise. That use we choose $\sigma=15, \sigma=25$ and $\sigma=50$ to conduct comparative experiments.

\section{Run Time:}

To appraise the concert metrics of image denoising methods PSNR is used as a representative quantitative measurement. Give a ground truth image $\mathrm{x}$, the PSNR of denoised images $\breve{\mathrm{x}}$ is defined by

Published By:

Blue Eyes Intelligence Engineering and Sciences Publication

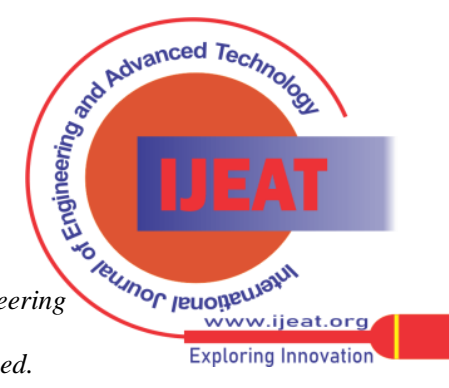


$\operatorname{PSNR}(\mathrm{x}, \breve{\mathrm{x}})=10 \cdot \log _{10}\left(\left\|x^{255}-x^{2}\right\|^{22}\right)$

PSNR and runtime of processing every image are two important factors of image denoising. The performance of the proposed methods has been verified in the gray image section. The PSNR is a different Dimension an image is tested for Grey images denoising as follows (table 2). We utilize noisy image sizes of $256 * 256$ and $512 * 512$ with different standard deviations such as $\sigma=15, \sigma=25$, and $\sigma=$ 50 to test the PSNR of different images for the Bilateral method. Specifically, we use Matlab-2019 to test the run process of Bilateral with CNN Table (3) we know that our Bilateral using CNN is modest with popular methods such as BM3D, WNNM, EPLL, CSF, TNRD, IRCNN, ECNDNet, and Bilateral with CNN.

\section{RESULT AND DISCUSSION}

We have taken various images for experimental purposes and we have seen that on different standards deviation such as 15,25 , and 50 obtained results have been mention below as under table 2 .

\section{Performance Evaluation}

According to the preceding research, we know that the denoising method can be articulated. It is being used to enhance the performance of the noise level and the increased PSNR. We consume noisy image sizes of

$256 * 256,512 * 512,1024 * 1024$ with $\sigma=15, \sigma=25$, and $\sigma=50$ to test the quickness of different methods for an image. Specifically, we use MATLAB to test CNN and bilateral filtering.

Our proposed method is a better PSNR for image denoising. We know that our bilateral filtering is competitive with popular methods such as BM3D, WNNM, EPLL, CSF, TNDR, and CNN. We will demonstrate the efficiency of these methods for image denoising. The performance of the recommended method has been proved the processing an image is tested for gray image denoising. I have accompanied some testing to see the performance of the proposed framework. I virtual noisy images by adding White Gaussian noise with various standard deviations to some standard test images. These noisy images were then denoised using several PSNR results were considered real images for each test image, three noisy versions were created by adding white Gaussian noise with standard deviations 15, 25, and 50.

\section{Results:}

Table 6.1 showing the different PSNR measuring on the different standard deviation and results finding the PSNR according to the different denoising techniques.

Table 6.1: Result of PSNR values different denoising method

\begin{tabular}{|l|c|c|c|c|l|l|}
\hline \multirow{2}{*}{ IMAGES } & \multicolumn{3}{|l|}{ Size of $\mathbf{2 5 6} * \mathbf{2 5 6}$} & \multicolumn{2}{l|}{ Size of $\mathbf{5 1 2} \mathbf{5 1 2}$} \\
\cline { 2 - 7 } & $\sigma=15$ & $\sigma=25$ & $\sigma=50$ & $\sigma=15$ & $\begin{array}{l}\sigma= \\
25\end{array}$ & $\sigma=50$ \\
\hline AIRPLANE & 34.88 & 34.61 & 34.04 & 36.03 & 35.93 & 35.72 \\
\hline BARBARA & 34.57 & 34.33 & 33.81 & 33.51 & 33.45 & 33.21 \\
\hline BOAT & 33.72 & 33.52 & 32.96 & 36.16 & 36.05 & 35.81 \\
\hline CAMRAMAN & 35.53 & 35.33 & 34.72 & 37.00 & 36.92 & 36.71 \\
\hline
\end{tabular}

\begin{tabular}{|l|c|c|c|c|c|c|}
\hline COUPLE & 33.61 & 33.44 & 32.82 & 34.02 & 33.95 & 33.70 \\
\hline LENA & 35.20 & 35.00 & 34.54 & 35.67 & 35.61 & 35.46 \\
\hline MAN & 33.65 & 33.44 & 32.87 & 34.35 & 34.25 & 33.98 \\
\hline MONARCH & 34.14 & 33.97 & 33.52 & 36.20 & 36.15 & 36.06 \\
\hline PEPPER & 35.67 & 35.51 & 35.01 & 34.05 & 33.98 & 33.85 \\
\hline STARFISH & 33.11 & 32.96 & 32.52 & 36.00 & 35.81 & 35.67 \\
\hline
\end{tabular}

Table 6.2 showing the different images data set measuring on the different standard deviation and results finding the peak Signal to noise ratio according to the database, where $\sigma$ is the noise level to different denoising images with different denoising techniques.

Table 6.2 Result of PSNR values different dimensions.

\begin{tabular}{|c|c|c|c|}
\hline METHODS & $\sigma=15$ & $\sigma=25$ & $\sigma=50$ \\
\hline BM3D & 32.37 & 29.97 & 26.72 \\
\hline WNNM & 32.70 & 30.26 & 27.05 \\
\hline EPLL & 32.14 & 29.69 & 26.47 \\
\hline CSF & 32.32 & 29.84 & - \\
\hline TNRD & 32.50 & 30.06 & 26.81 \\
\hline IRCNN & 32.77 & 30.38 & 27.14 \\
\hline ECNDNet & 32.81 & 30.39 & 27.15 \\
\hline Bilateral with & 34.73 & 34.50 & 33.98 \\
CNN & & & \\
\hline
\end{tabular}

Table 6.3 showing the different accuracy measuring on the different standard deviation of different images for bilateral filtering and results finding the better PSNR according to the database.

Table 6. 3: Result of the previous result and current result on different standard deviation.

\begin{tabular}{|c|c|c|c|c|c|c|}
\hline \multirow{2}{*}{ IMAGES } & \multicolumn{3}{|c|}{$\begin{array}{l}\text { Previous } \\
\text { result }\end{array}$} & \multicolumn{3}{|c|}{ Current result } \\
\hline & $\sigma=15$ & $\sigma=25$ & $\sigma=50$ & $\sigma=15$ & $\sigma=25$ & $\sigma=50$ \\
\hline BOAT & 32.37 & 30.14 & 27.16 & 33.71 & 33.49 & 32.98 \\
\hline PEPPERS & 33.25 & 30.85 & 27.30 & 35.63 & 35.49 & 34.21 \\
\hline STARFISH & 32.17 & 29.43 & 25.72 & 33.63 & 33.47 & 33.18 \\
\hline MONARCH & 33.11 & 30.30 & 26.82 & 34.12 & 34.07 & 33.47 \\
\hline LENA & 34.52 & 32.38 & 29.29 & 35.17 & 35.08 & 34.42 \\
\hline MAN & 32.39 & 30.03 & 30.33 & 33.58 & 33.45 & 32.85 \\
\hline COUPLE & 32.39 & 30.03 & 26.84 & 33.62 & 33.41 & 32.88 \\
\hline
\end{tabular}

Blue Eyes Intelligence Engineering and Sciences Publication

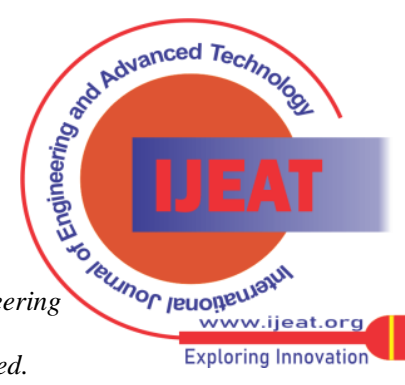



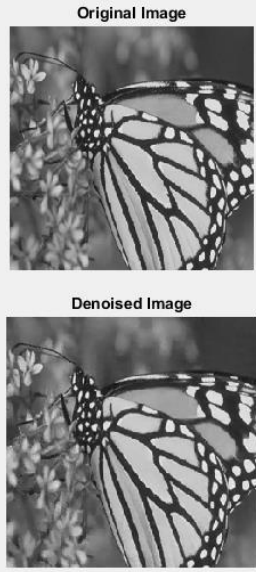
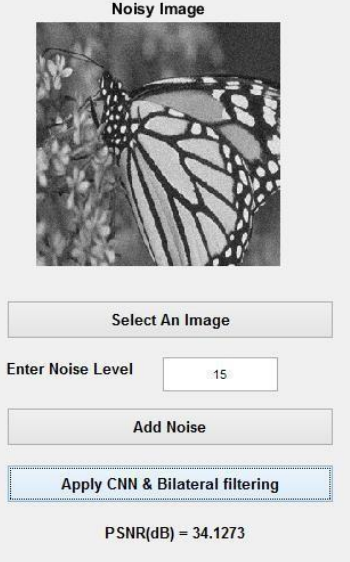

Figure 6.1: PSNR values bilateral methods using CNN technique noise level 15.
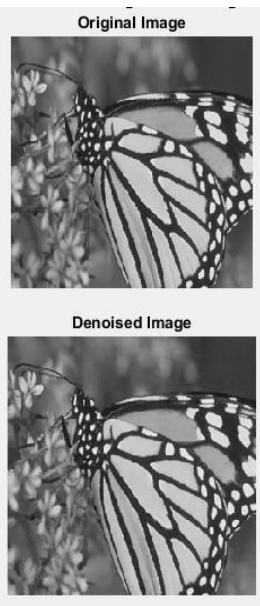

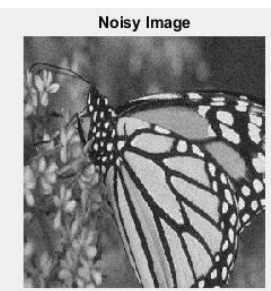

Select An Image

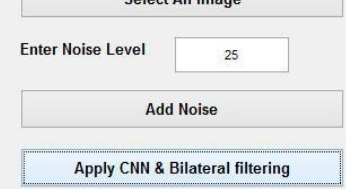

$\operatorname{PSNR}(\mathrm{dB})=34.072$

Figure 6.2: PSNR values bilateral methods using CNN technique noise level 25.

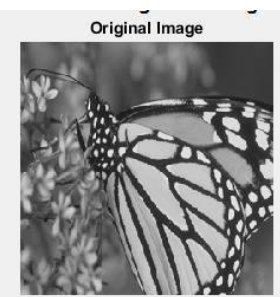

Denoised Image

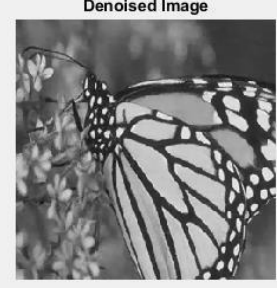

Noisy Image

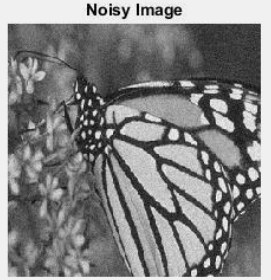

Select An Image

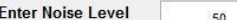

Enter Noise Level $\quad 50$

Add Noise

Apply CNN \& Bilateral filtering

$\operatorname{PSNR}(\mathrm{dB})=33.4755$

Figure 6 .3: PSNR values bilateral methods u sing CNN technique noise level 50

Figure 6.1 represents the image denoising technique in which an original image becomes a noisy image by adding Gaussian noise up to level 15 and then applying by both technique CNN and Bilateral filtering, which offers

As 34.12(dB) PSNR likewise in fig2 and fig3 (standards deviation) on the noise level 25 and 50 use does the same experimental for the image denoising and we get $34.07(\mathrm{~dB})$ and 33.47 respectively.

\section{SCOPE}

Future expansion of this approach is to be with proposed technique the proposed filtering method work very properly for noise care of different filtering approach. We will combine model-based Optimization and

Discriminative gaining knowledge of strategies to get rid of the noise from the real noise images. For future work, first, explore how to covenant with different sorts of noise mainly these current in actual life. Secondly, the technique Besides the methodology of pictures denoising can also be improved in two other applications.

\section{CONCLUSION}

This paper compares the most widely used image denoising different techniques and it concludes that based on the data set we have to find the best performance and increasing PSNR value for the bilateral filter using the CNN technique, for image denoising. We have to find a better PSNR value for the image denoising technique.

\section{REFERENCE}

1. V. Aurich and J.Weule, "Non-linear Gaussian filters performing edge preserving diffusion," in Proceedings of the DAGM Symposium, pp. 538-545, 1995.

2. C. Tomasi and R. Manduchi, "Bilateral filtering for gray and color images," in Proceeding of the IEEE International Conference, pp.839-846.1998

3. D.Barash, "A fundamental relationship between bilateral filtering, adaptive smoothing, and the nonlinear diffusion equation," IEEE Transaction, vol. 24, no. 6, pp. 844-847, 2002.

4. S. Paris, F. Durand, "A fast approximation of the bilateral filter using a signal processing approach", Proc. European Conference on Computer Vision, 2006.

5. F.porkli, "Constant Time O (1) Bilateral Filtering", IEEE Computer Society Conference on Computer Vision and Pattern Recognition(CVPR), July 2008.

6. G. Goyal, "Impact and analysis of improved bilateral filter on TEM images" International Journals of Science and research, vol.3, no.6(2014)

7. ShuhangGU; Lei Zhang, "Weighted Nuclear Norm Minimization with application to image denoising" IEEE Conference on Computer Vision and Pattern Recognition. DOI: 10.1109/CVPR.2014.36625 September 2014

8. Jeremias Sulam and Michael Elad, "Expected Patch Log Likelihood with a Sparse Prior" Springe International Publishing Switzerland, LNCS 8932, pp. 99111, 2015

9. Zhang, Ren, et al. "Deep residual learning for image recognition. IEEE, pp. 770-778 June 2016

10. Yunjin Chen and Thomas Pock, " Trainable Nonlinear ReactionDiffusion: A Flexible Framework for

Fast and Effective Image Restoration" IEEE transaction vol.XX, no.XX, 2016

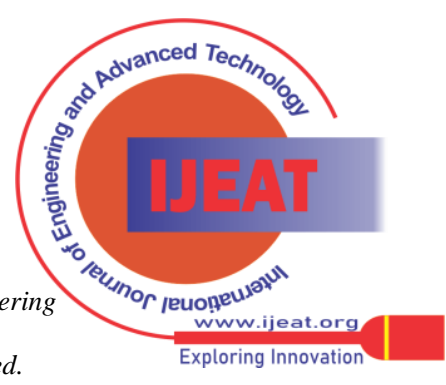


11. K. Zhang, W. Zuo, Y. Chen, D. Meng, and L. Zhang, "Beyond a Gaussian denoiser: Residual learning of deep CNN for image denoising," IEEE Transactions on

Image Processing, vol. 26, no. 7, pp. 3142-3155, July 2017.

12. Bako, S., Vogels, T., McWilliams, B., et al.: 'Kernelpredicting convolutional networks for denoising Monte Carlo renderings', ACM Trans. Graph, 2017, 36, (4), pp. 1-14

13. Ahn, B., Cho, N.I.: 'Block-matching convolutional neural network for image denoising', arXiv preprint arXiv:1704.00524, 2017

14. N.Senthilkumaran [1], J.Saromary [2], Detailed Performance Evaluation of Bilateral Filters for De-noising Chromosome Image Assistant Professor [1], M.phil. Research Scholar [2] Department of Computer Science and Applications. Gandhi gram Rural Institute Deemed University Ganthigram-624302, Dindigul Tamil Nadu India International Journal of Information Technology (IJIT) Volume 3 Issue 3, May-Jun 2017

15. Tobias Plötz Stefan Roth "Neural Nearest Neighbors Networks" Department of Computer Science, TU Darmstadt. 1810.12575v1, 30 OCT 2018.

16. Kai Zhang, WangmengZuo, Senior Member, IEEE, and Lei Zhang FFD Net: Toward a Fast and Flexible Solution for CNN based Image Denoising IEEE. 1710.04026v2, 22 may 2018.

17. Liu, P., Zhang, H., Zhang, K., et al.: 'Multi-level wavelet-CNN for image restoration', arXiv preprint arXiv: 1805.07071, 2018

18. Qingbowu, wen qi Ren, Xiaochun Cao" Learning interleaved cascade of Shrinkage field for denoising": IEEE transaction image processing volume 29 DOI: 10.1109/TIP.2019.2942504

19. Enhanced CNN for image denoising Chunwei Tian1,2, Yong Xu1,2 $\square$, Lunke Fei3, Jungian Wang1,2, Jie Wen1,2, Nan Luo4, BioComputing Research Center,Harbin Institute of Technology, Shenzhen, ISSN 24682322 Received on 8th November 2018 Revised on 27th December 2018 Accepted on 7th January 2019 DOI:

10.1049/trit.2018.1054 www.ietdl.org.

\section{AUTHORS PROFILE}

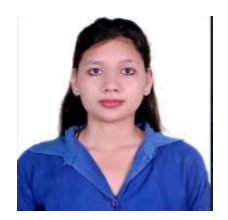

Pallavi Bora D/of Bhagwan Singh Bora, M.Tech scholar (with specialization in Computer science Engineering) from B.T.K.I.T DwarahatAlmora Uttarakhand. (263653)DOB 30 june1994

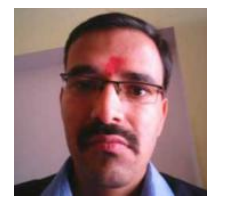

Dr. Kapil Chaudhary, Received the M.Tech degree with a specialization in Digital Signal Processing from the G.B Pant Institute of Engineering\&Technology and the Ph.D. degree with Prof.B.K Kaushik (Assoc Prof IIT Roorkee) in Computer Science\&Engineering with specialization in image denoising from the Himalyan University with Prof. Narender Kumar(Asst Prof HNBGU), in 2008 and 2016, respectively. He joined the Computer Science EngineeringDepartment, BTKIT, Dwarahat, research interests include image processing, image denoising, Infrared image capture.

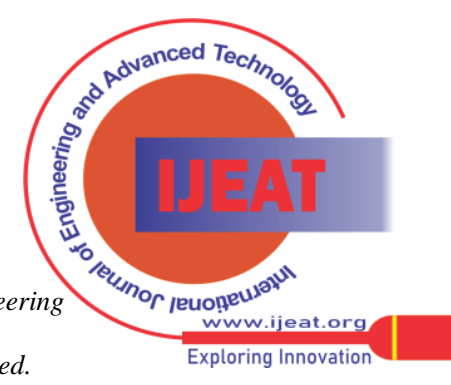

